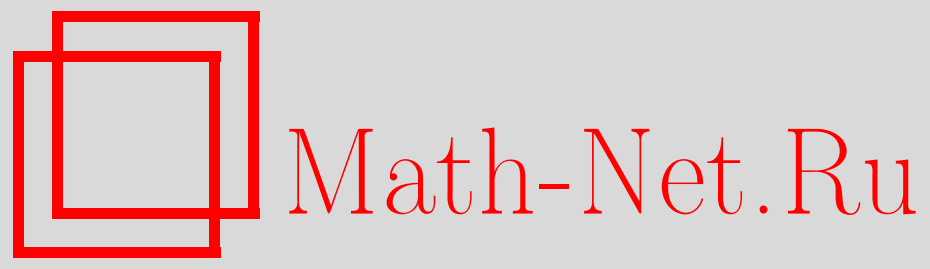

С. А. Назаров, Я. Таскинен, О спектре третьей краевой задачи в области с пиком, Функи. анализ и его прил., 2011, том 45, выпуск 1, 93-96

DOI: https://doi.org/10.4213/faa3020

Использование Общероссийского математического портала Math$\mathrm{Net.Ru} \mathrm{подразумевает,} \mathrm{что} \mathrm{вы} \mathrm{прочитали} \mathrm{и} \mathrm{согласны} \mathrm{с} \mathrm{пользователь-}$ ским соглашением

http://www.mathnet.ru/rus/agreement

Параметры загрузки:

IP : 35.173 .219 .12

26 апреля 2023 г., 13:42:21

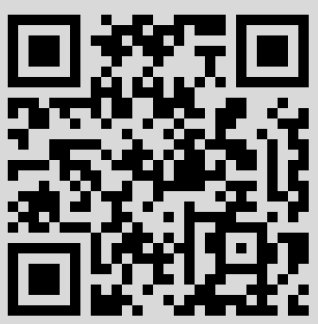




\title{
ЛитерАТУРА
}

[1] М. Холл, Комбинаторика, Мир, М., 1970. [2] Ж. Лаллеман, Полугруппъ и комбинаторные приложения, Мир, М., 1985. [3] Е. А. Горин, Формулы обращения Чебышева-Мёбиуса в контексте абелевых полугрупп, в кн.: IV Международная конференция по теории чисел и прил., Тула (2001), Тезисы докл., Изд-во МГУ, 2001, 48-49. [4] А. Клиффорд, Г. Престон, Алгебраическая теория полугрупп, т. I, Мир, M., 1972.

Московский педагогический государственный университет, кафедра математического анализа

Поступило в редакцию e-mail: evgeny.gorin@mtu-net.ru

\section{О спектре третьей краевой задачи в области с пиком*}

\author{
(c) 2011. С. А. НАзАров, Я. ТАСКИНЕН
}

Памяти М. Ш. Бирмана посвящается

Пусть $\omega$ - область в евклидовом пространстве $\mathbb{R}^{n-1}$ с липшицевой границей $\partial \omega$ и компактным замыканием $\bar{\omega}=\omega \cup \partial \omega$. Введем пик высоты $d>0$

$$
\Pi_{d}=\left\{x=(y, z) \in \mathbb{R}^{n-1} \times \mathbb{R}: z \in(0, d), z^{-1-\gamma} y \in \omega\right\}
$$

и область $\Omega$, совпадающую с пиком $\Pi_{d}$ внутри цилиндра $\{x:|y|<R, z \in(0, d)\}$, причем $R>0, \bar{\Omega}$ - компакт, $\gamma \geqslant 0$ - показатель заострения, а граница $\Gamma=$ $\partial \Omega$ заведомо липшицева всюду, кроме вершины пика $\mathscr{O}$. Рассмотрим третью краевую спектральную задачу в вариационной постановке

$$
(\nabla u, \nabla v)_{\Omega}+(b u, v)_{\Gamma}=\lambda(u, v)_{\Omega}, \quad v \in H^{1}(\Omega) \cap L_{2}(\Gamma),
$$

где $\nabla$ - градиент, $(,)_{\Omega}$ - скалярное произведение в пространстве Лебега $L_{2}(\Omega), \lambda$ - спектральный параметр, а $b$ - вещественная измеримая ограниченная функция на $\Gamma$, удовлетворяющая при некотором $b_{0} \in \mathbb{R}$ соотношению

$$
\left|b(x)-b_{0}\right| \leqslant c_{b}|x|^{\alpha} \quad \text { при п. в. } x \in \Gamma, \alpha>0 .
$$

Если $\gamma=0$, то $\Pi_{d}-$ конус, $\Gamma$ - липшицева поверхность и ввиду компактности вложений пространства Соболева $H^{1}(\Omega)$ в $L_{2}(\Omega)$ и $L_{2}(\Gamma)$ билинейная форма $q(u, v)=(\nabla u, \nabla v)_{\Omega}+(b u, v)_{\Gamma}$ в левой части формулы $(2)$ замкнута в $H^{1}(\Omega)$ и полуограничена, а значит, задаче (2) ставится [1, гл. 10] в соответствие самосопряженный оператор $A$ с дискретным спектром, образующим неограниченную последовательность собственных чисел

$$
\lambda_{1} \leqslant \lambda_{2} \leqslant \ldots \leqslant \lambda_{k} \leqslant \ldots
$$

указанных с учетом кратностей. При $\gamma>0$ граница $\Gamma$ перестает быть липшицевой в точке $\mathscr{O}$, а вложения $H^{1}(\Omega) \subset L_{2}(\Gamma)$ может не быть (ср. [2], [3] и

*Работа выполнена при финансовой поддержке Российского фонда фундаментальных исследований (проект 09-01-00759) и гранта № 127245 Академии Финляндии. 
др.). Однако в случае $b_{0}>0$ форма $q$ остается полуограниченной и порождает самосопряженный оператор $A$ со спектром (4). Согласно весовому неравенству

$$
\left\||x|^{-1} u ; L_{2}(\Omega)\right\|+\left\||x|^{(\gamma-1) / 2} u ; L_{2}(\Gamma)\right\| \leqslant c\left\|u ; H^{1}(\Omega)\right\|
$$

(см., например, [4]), вложение $H^{1}(\Omega) \subset L_{2}(\Gamma)$ компактно при $\gamma \in(0,1)$. В [4] проверено, что при $\gamma=1$ это вложение не является компактным, а при $\gamma>1$ оно вообще неверно. Точно так же можно убедиться в том, что для $b_{0}<0$ и $\gamma>1$ форма $q$ перестает быть полуограниченной. Цель данного сообщения описать спектр задачи (2) в межевом случае $\gamma=1$, для которого форма $q$ перестает быть полуограниченной при условии, что

$$
b_{0} \leqslant-t_{n}(\omega), \quad t_{n}(\omega):=(n-3 / 2)^{2}\left(\operatorname{mes}_{n-2} \partial \omega\right)^{-1} \operatorname{mes}_{n-1} \omega .
$$

Полуограниченность формы $q$ при $\gamma=1$ и $b_{0}>-t_{n}(\omega)$ вытекает из оценки [4]

$$
\left\|u ; L_{2}(\Gamma)\right\|^{2} \leqslant\left(t_{n}(\omega)-\varepsilon\right)^{-1}\left\|\nabla u ; L_{2}(\Omega)\right\|^{2}+C_{\varepsilon}\left\|u ; L_{2}(\Omega)\right\|^{2},
$$

где $\varepsilon>0$ и $C_{\varepsilon} \rightarrow+\infty$ при $\varepsilon \rightarrow+0$. Поэтому выше «порога» $-t_{n}(\omega)<0$ форма определяет самосопряженный оператор $A$ с чисто дискретным спектром (4).

В ситуации (5) дифференциальным выражением оператора $A$ задачи (2) является оператор Лапласа $-\Delta$ (со знаком минус), а областью определения линеал

$$
\begin{aligned}
\mathscr{D}(A)=\{u \in & H^{1}(\Omega): \Delta u \in L_{2}(\Omega), \\
& \left.(\nabla u, \nabla v)_{\Omega}+(b u, v)_{\Gamma}=(-\Delta u, v)_{\Omega}, v \in C_{c}^{\infty}(\bar{\Omega} \backslash \mathscr{O})\right\} \subset L_{2}(\Omega) .
\end{aligned}
$$

Отметим, что краевое условие $\partial_{\nu} u+b u=0$ «спрятано»в интегральном тождестве из определения (6) и выполнено для гладких функций $u$ из $\mathscr{D}(A)$, так как дифференцирование $\partial_{\nu}$ вдоль внешней нормали определено почти всюду на липшицевой поверхности $\Gamma \backslash \mathscr{O}$. В случае полуограниченной формы $q$ (см. выше) порожденный ею оператор $A$ обладает такой же областью определения (6).

Изучение спектра $\sigma(A)$ основано на утверждениях о разрешимости задачи

$$
(\nabla u, \nabla v)_{\Omega}+(b u, v)_{\Gamma}=f(v), \quad v \in V_{-\beta}^{1}(\Omega),
$$

где $\beta \in \mathbb{R}$ - весовой индекс, $V_{\beta}^{1}(\Omega)$ - пространство Кондратьева с нормой

$$
\left\|u ; V_{\beta}^{1}(\Omega)\right\|=\left(\left\||x|^{\beta} \nabla u ; L_{2}(\Omega)\right\|^{2}+\left\||x|^{\beta-1} u ; L_{2}(\Omega)\right\|^{2}\right)^{1 / 2},
$$

$f \in V_{-\beta}^{1}(\Omega)^{*}$ - линейный непрерывный функционал на пространстве $V_{-\beta}^{1}(\Omega)$, а решение $u$ ищется в классе $V_{\beta}^{1}(\Omega)$.

Лемма 1. Пусть $\gamma=1$ и выполнено условие (5). Оператор $\mathscr{A}_{\beta}: V_{\beta}^{1}(\Omega) \rightarrow$ $V_{-\beta}^{1}(\Omega)^{*}$ задачи (7) фредгольмов в том и только в том случае, когда $\beta \neq 0$.

Замечание. Если $b_{0}>-t_{n}(\omega)$, то оператор $\mathscr{A}_{0}: V_{0}^{1}(\Omega) \rightarrow V_{0}^{1}(\Omega)^{*}$ фредгольмов, однако при условии (5) его образ незамкнут. Именно этим и обусловлено обнаруженное в работе различие в строении спектра $\sigma(A)$ выше и ниже порога.

Для более точного описания области определения (6) оператора $A$ понадобится асимптотическое разложение решения $u(x)$ около точки $\mathscr{O}$. Членами разложения служат решения

$$
\begin{gathered}
U_{ \pm}(z)=z^{\Lambda_{ \pm}}, \quad \Lambda_{ \pm}=-(n-3 / 2)\left(1 \pm i \sqrt{-b_{0} t_{n}(\omega)^{-1}-1}\right), \quad \text { при } b_{0}<-t_{n}(\omega), \\
U_{ \pm}(z)=2^{-1 / 2} z^{-n+3 / 2}(1 \pm i \ln z) \quad \text { при } b_{0}=-t_{n}(\omega)
\end{gathered}
$$


обыкновенного дифференциального уравнения эйлеровского типа

$$
-\frac{\partial}{\partial z} z^{2(n-1)} \frac{\partial U}{\partial z}(z)-b_{0} \frac{\operatorname{mes}_{n-2}(\partial \omega)}{\operatorname{mes}_{n-1}(\omega)} z^{2(n-2)} U(z)=0, \quad z>0
$$

полученного в [4] путем формального асимптотического анализа задачи (2).

Лемма 2. Пусть $\gamma=1$ и выполнено условие (5). Пусть, кроме того, $\beta>$ $\beta^{\sharp} \geqslant \beta-\alpha, \beta, \beta^{\sharp} \neq 0, f \in V_{-\beta^{\sharp}}^{1}(\Omega)^{*} \subset V_{-\beta}^{1}(\Omega)^{*}$, a $u \in V_{\beta}^{1}(\Omega)-$ решение задачи (7). Тогда

$$
u(x)=\chi(x)\left(C_{+} U_{+}(z)+C_{-} U_{-}(z)\right)+\widetilde{u}(x)
$$

где х - гладкая срезающая функиия с носителем в $\overline{\Pi_{d}}$, равная единице вблизи точки $\mathscr{O}, \widetilde{u} \in V_{\beta^{\sharp}}^{1}(\Omega), C_{ \pm} \in \mathbb{C}$, но $C_{ \pm}=0$ в случае $0 \notin\left(\beta^{\sharp}, \beta\right)$. Имеет место оценка

$$
\left|C_{+}\right|+\left|C_{-}\right|+\left\|\widetilde{u} ; V_{\beta^{\sharp}}^{1}(\Omega)\right\| \leqslant c_{\beta, \beta^{\sharp}}\left(\left\|u ; V_{\beta}^{1}(\Omega)\right\|+\left\|f ; V_{\beta}^{1}(\Omega)^{*}\right\|\right) .
$$

Согласно (9), решения задачи (7) в классах $V_{\beta}^{1}(\Omega)$ и $V_{-\beta}^{1}(\Omega)$ при $\beta \in(0, \alpha)$ различаются двумя асимптотическими слагаемыми (8), и из леммы 2 , примененной двукратно (с парами показателей $\{\beta, \delta\}$ и $\{\delta,-\beta\} ;$ здесь $0<\delta<$ $\min \{\beta, \alpha-\beta\})$, выводится связь Ind $\mathscr{A}_{\beta}=$ Ind $\mathscr{A}_{-\beta}+2$ индексов операторов $\mathscr{A}_{\beta}$ и $\mathscr{A}_{-\beta}$, которые являются взаимно сопряженными. В итоге Ind $\mathscr{A}_{\beta}=-$ Ind $\mathscr{A}_{-\beta}=1$. Леммы 1 и 2 вместе с этой формулой влекут за собой следующие утверждения.

Теорема 1. Пусть $\gamma=1$ и выполнено условие (5).

1. Функиия и из области определения (6) оператора А принадлежит пространству $V_{-1}^{1}(\Omega)$, более узкому, чем исходное пространство $H^{1}(\Omega)$.

2. Oператор $A$ замкнут и симметричен, а его сопряженный $A^{*}$ имеет область определения $\mathscr{D}\left(A^{*}\right)=\left\{u: u-\chi\left(C_{+} U_{+}+C_{-} U_{-}\right) \in V_{-\beta}^{1}(\Omega), \beta \in(0, \alpha)\right.$, $\left.(\nabla u, \nabla v)_{\Omega}+(b u, v)_{\Gamma}=(-\Delta u, v)_{\Omega}, v \in C_{c}^{\infty}(\bar{\Omega} \backslash \mathscr{O})\right\} \subset V_{1}^{1}(\Omega) \subset L_{2}(\Omega)$.

3. Точечный спектр оператора $A^{*}$ и остаточный спектр оператора $A$ занимают всю комплексную плоскость $\mathbb{C}$. Любая точка $\lambda \in \mathbb{C}$, за исключением счетного набора $\Sigma$ вещественных чисел, - простое собственное число оператора $A^{*}$. Кратности собственных чисел из $\Sigma$ конечны.

Теорема 1, в частности, показывает, что симметрический оператор $A$ не является самосопряженным, но в силу теоремы 1 его индексы дефекта равны 1 , 1 и, следовательно, $A$ допускает самосопряженные расширения.

Теорема 2. Самосопряженные расширения $A(\varphi)$ оператора $A$ параметризуются переменной $\varphi \in[0,2 \pi)$. Области определения имеют вид

$$
\mathscr{D}(A(\varphi))=\left\{u \in \mathscr{D}\left(A^{*}\right): C_{+}=e^{i \varphi} C_{-}\right\} .
$$

Вложение $\mathscr{D}(A(\varphi)) \subset L_{2}(\Omega)$ компактно, а значит, спектр оператора $A(\varphi)$ дискретный и вещественный. В связи с отсутствием свойства полуограниченности у формы $q$ этот спектр формирует две неограниченные последовательности

$$
0 \leqslant \lambda_{1}(\varphi) \leqslant \cdots \leqslant \lambda_{k}(\varphi) \leqslant \cdots \quad \text { и } \quad 0>\lambda_{-1}(\varphi) \geqslant \cdots \geqslant \lambda_{-k}(\varphi) \geqslant \cdots .
$$


Итак, при условии (5), т.е. ниже порога, оператор третьей краевой задачи с областью определения (6) несамосопряжен и никакое его самосопряженное расширение $A(\varphi)$ не является полуограниченным (см. (11)). Какого-либо канонического способа выбора области определения (10) расширения не обнаружено.

\section{ЛитЕРАТУРА}

[1] М. Ш. Бирман, М. З. Соломяк, Спектралъная теория самосопряженных операторов в гильбертовом пространстве, изд-во Ленингр. ун-та, Л., 1980. [2] В. Г. Мазья, Пространства Соболева, изд-во Ленингр. ун-та, Л., 1985. [3] D. Daners, Trans. Amer. Math. Soc., 352:9 (2000), 4207-4236. [4] С. А. Назаров, Я. Таскинен, Вестник СПбГУ, cep. 1, 2008, № 1, 56-65.

Институт проблем машиноведения РАН

Поступило в редакцию e-mail: srgnazarov@yahoo.co.uk 19 августа 2009 г.

University of Helsinki

e-mail: jari.taskinen@helsinki.fi

Заведующая редакцией и научный редактор Г. М. Цукерман

Сдано в набор 24.12.2010. П Подписано к печати 24.1.2011. Формат 70×100/16

Печать офсетная. Усл. печ. л. 7,8. Усл. кр.-отт. 1,8 тыс. Бум. л. 3,0

Уч.-изд. л. 8,0. Тираж 218 экз. Заказ 993.

Учредитель: Российская академия наук

Адрес редакции: 117966 Москва, ГСП-1, ул. Губкина 8, комн. 624. Тел. 938-37-56

Издатель: Российская академия наук, Издательство «Наука»,

117997 Москва, Профсоюзная, ул. 90

Отпечатано в ППП «Типография «Наука», 121099 Москва, Шубинский пер., 6 